\title{
Alterations in elastin of the optic nerve head in human and experimental glaucoma
}

\author{
Harry A Quigley, Andrew Brown, Mary Ellen Dorman-Pease
}

\begin{abstract}
The optic nerve heads from normal and glaucomatous eyes of humans and monkeys were examined by light and electron microscopy for the presence and distribution of elastin. Elastin densely lined the insertion of the lamina cribrosa into the sclera and was prominent in the laminar beams. The long axis of elastin paralleled that of the collagen fibrils and corresponded to the directions of expected forces on the tissue. In glaucomatous eyes elastin molecules were curled instead of straight and seemed disconnected from the other elements of the connective tissue matrix. Laminar beams stretch and reorganise their substructure during glaucomatous atrophy, probably leading to changed compliance. Differences in elastin function may have a part in susceptibility to glaucomatous injury.
\end{abstract}

Glaucoma is best defined as an optic neuropathy associated with a characteristic excavation of the optic disc ${ }^{1}$ and a progressive loss of visual field sensitivity. This neuropathy is related to the level of the intraocular pressure (IOP) such that, the higher the IOP the more likely the neuropathy is to occur. ${ }^{2}$ In some persons it develops at what is considered normal IOP, while in many others the IOP is intermittently or consistently above the statistically normal level.

The IOP acts on the optic nerve head and the sclera by two force vectors. Firstly, the IOP is usually higher than optic nerve tissue pressure, generating an inside-out force. Secondly, the IOP generates a wall tension that pulls on the perimeter of the nerve head. The tissues at this complex opening in the eye wall are specialised to account for these forces. ${ }^{3-5}$ This specialised composition allows the safe passage of nerve fibres out of the eye and the maintenance of an appropriate blood supply to them under normal conditions.

We have conducted detailed studies of the extracellular connective tissues of the optic nerve head. Collagen, glycosaminoglycans, and elastin are instrumental in determining the response of the eye to the forces produced by IOP. Anderson ${ }^{6}$ may have been the first to describe the presence of elastin within the lamina cribrosa by electron microscopy. We here demonstrate the detailed distribution of elastin in the normal optic nerve head by electron microscopy and by a histochemical method not previously used in ocular tissues. These techniques illustrate how elastin may contribute to the biomechanical response of the nerve head. Features of extracellular structure may have an important role in determining the susceptibility of the individual eye to pressure-induced forces. In addition we present the eyes of human eye bank donors who had had glaucoma and the eyes of monkeys with experimental glaucoma. The appearance of elastin fibres and the surrounding connective tissue elements are dramatically changed by the IOP.

\section{Material and methods}

We studied 10 human eyes that had no history of eye disease and 19 human glaucomatous eyes with a history of raised IOP and/or clinical glaucomatous damage. Only one eye per person was examined. Control eyes had a normal neural area in their optic nerve cross-section, which was embedded in epoxy resin, thick-sectioned at $1 \mu \mathrm{m}$, and analysed..$^{78}$ Normal and glaucomatous donors did not differ in age (mean $=74$ years in each group, $\mathrm{p}>0.05$ ); $90 \%$ of both donor groups were Caucasian, and the two groups were equally divided between men and women.

Human glaucomatous eyes were fixed in buffered aldehydes within 24 hours of death. The degree of glaucomatous damage was determined by the visual field test result, the optic nerve neural area and/or fibre count, or both (Table 1). Nineteen glaucomatous eyes were studied by light microscopy, at least three from each of the five severity stages. In addition we examined by electron microscopy six normal and seven glaucomatous human eyes, two each from stages 1,4 , and 5 and one from stage 3 .

In cynomolgus monkeys (Macaca fascicularis) we caused a raised IOP in one eye by laser trabecular treatment. ${ }^{9}$ This produces an optic neuropathy that is indistinguishable from human glaucoma in clinical and histopathological details. The animals were anaesthetised with intravenous pentobarbitone, killed by exsanguination, the eyes fixed by vascular perfusion of aldehyde fixatives, and the optic nerve heads of their damaged and normal eyes dissected as described below. We studied the nerve heads of five glaucomatous monkey eyes and their fellow eye controls. The degree of injury in monkey eyes was judged by the degree of fibre loss in the optic nerve cross sections in similar fashion to human eyes. Two of the monkey eyes had stage 3 damage and three had stage 5

Table 1 Criteria for severity staging of human glaucoma eyes

\begin{tabular}{llc}
\hline & Visual field & Optic nerve area \\
\hline Stage $1^{\star}$ & Normal & $>85 \%$ of normal \\
Stage 2 & Normal & $75-85 \%$ of normal \\
Stage 3 & Usually abnormal & $50-75 \%$ of normal \\
Stage 4 & Abnormal & $25-50 \%$ of normal \\
Stage 5 & Advanced loss & $<25 \%$ of normal \\
\hline
\end{tabular}

In seven of the 19 eyes only one of the two parameters was available.

^ Stage 1 consists of eyes suspected of being glaucomatous that had statistically abnormal intraocular pressure. 
damage. This research complied with the ARVO Resolution on Animals and was approved and monitored by the Johns Hopkins Animal Care Committee.

For light microscopy we removed the optic nerve head and trimmed the surrounding sclera to identify the superior position. The tissue was rinsed in phosphate buffer, and the retina was removed. Nerve heads were placed in 30\% sucrose in distilled water for cryoprotection, frozen in liquid nitrogen, and serially sectioned at $10 \mu \mathrm{m}$ thickness parallel to the interface of the nerve head with the vitreous cavity. This method gave similar tissue preservation to paraffin embedding. Frozen, unfixed sections were stained by Luna's method, whose major steps are: (1) 30 minutes in aldehyde fuchsin; (2) alcohol rinse; and (3) 1 minute in haematoxylin to stain the alpha elastin fibres. ${ }^{10}$ By staining alternate sections we found the Luna technique to be superior to Verhoeff-van Gieson and other histochemical methods for highlighting the three-dimensional elastin network. We confirmed by electron microscopic methods described below that the position and relative number of stained elements with the Luna method correspond to alpha elastin. In addition our previous light microscopic studies of staining of alpha elastin by antibodies labelled by the immunoperoxidase method indicate that the distribution of stained material is similar to that appearing in cross section. $B$. Collagen $(C)$ and elastin (arrows) are both in longitudinal section. (Normal monkey nerve head, $\times 17000$.)
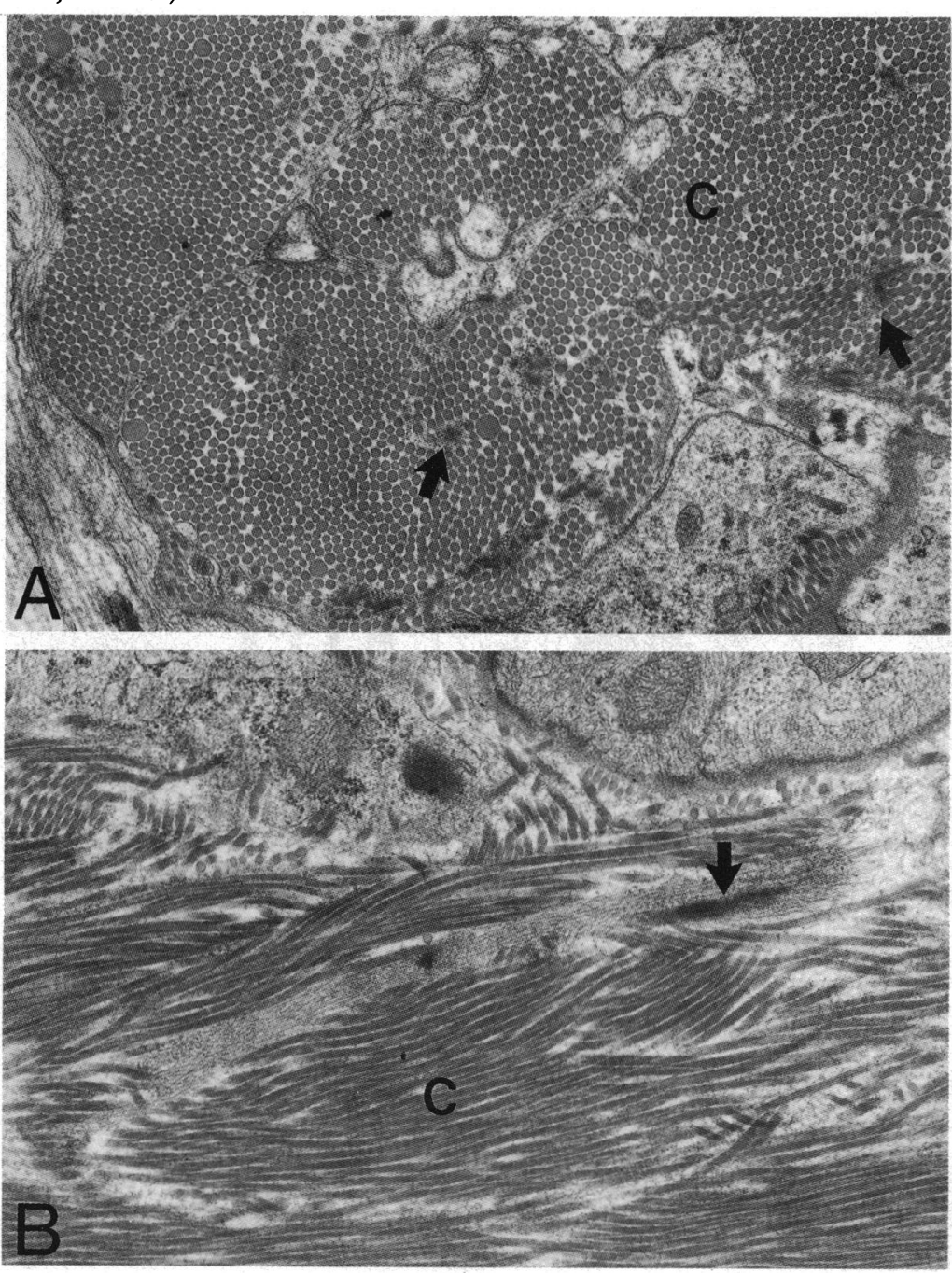

obtained with the Luna method. The nerve heads were analysed by region in the normal eyes (superior, inferior, nasal, temporal). In the glaucomatous eyes the degree of nerve fibre loss within an optic nerve cross section ${ }^{78}$ was correlated with the appearance of elastin in each region. The findings in human and monkey eyes were similar, but only human examples are presented here.

For electron microscopy nerve heads were postfixed in $1 \%$ osmium tetroxide and stained with tannic acid, uranyl acetate, and lead citrate after thin sectioning, to define better the alpha elastin. ${ }^{1}$ We have demonstrated previously that the elements referred to as elastin fibres ${ }^{12}$ stain with antibodies to alpha elastin. ${ }^{13}$

\section{Results}

Our normal material provided an important comparison with the glaucomatous tissues prepared by the same methods. There was a large amount of elastin in the optic nerve head region. The specificity of the Luna stain was confirmed by staining of Bruch's membrane and arteriolar walls. The peripapillary sclera had more elastin than the sclera distant from the nerve. By electron microscopy the long axes of elastin fibres were parallel to the orientation of the collagen bundles in which they resided (Fig 1). Elastin stained darkly with tannic acid, and the specificity of this staining had been previously confirmed by labelling with antibodies against alpha elastin conjugated with gold particles (Fig 2).

In the immediate $200-300 \mu \mathrm{m}$ zone of insertion of the lamina cribrosa, elastin fibrils ringed the nerve head in a dense band (Fig 3). From this ring elastin fibrils originated at nearly a right angle, entering the base of each beam of the lamina cribrosa. Each laminar beam had several elastin fibres, with collagen spaced between them (Fig 4). The elastin fibres in the normal human or monkey lamina were invariably straight in appearance, bending only slightly as they passed from one beam to another. There were only minimal differences between regions of the nerve head cross section in elastin distribution. However, anteroposteriorly elastin density was highest at the level of the sclera and lower at the level of the retina and posterior to the line of myelination (Fig 5).

In the stage 1 glaucomatous eyes no definite differences from the normal pattern of elastin staining were observed with light or electron microscopy. In all the stage 2 nerve heads the appearance of elastin was abnormal. In some beams the normally straight elastin molecules appeared curvilinear, like small sine waves (Fig 4). This was most prominent in atrophic zones of the nerve. At damage stages 3 and 4 every eye had curved elastin profiles in many beams. In eyes with severe damage (stage 5 ) the interpretation of the findings was made difficult by the extreme change in laminar architecture.

We correlated the sectors of substantial neural loss with the presence of curled elastin. Six eyes were not included in this analysis because their damage was uniform. Moreover, we excluded the four stage 5 eyes that had nearly total loss of fibres. In five of the remaining nine eyes the most 
Figure 2 Collagen and elastin from normal monkey lamina cribrosa (upper left) shows the normally close apposition of collagen fibrils to the microfibrillar component of the elastin complex (arrows). The upper right micrograph from a normal human lamina shows that the darkly stained core (white $E$ in upper left) is labelled by antibodies against alpha elastin that were conjugated with $15 \mathrm{~nm}$ colloidal gold particles. ${ }^{4}$ The two lower micrographs are from lamina of monkeys with experimental glaucoma.

Note the much greater separation between elastin's microfibrils (arrows), compared with the normal appearance upper left. (The separation present in the gold-labelled specimen, upper right, is due to preparation conditions necessary for immunoelectron microscopy.) (Upper left lower right and left: monkey, $\times 48000$; upper right. normal human, $\times 37000$ ).
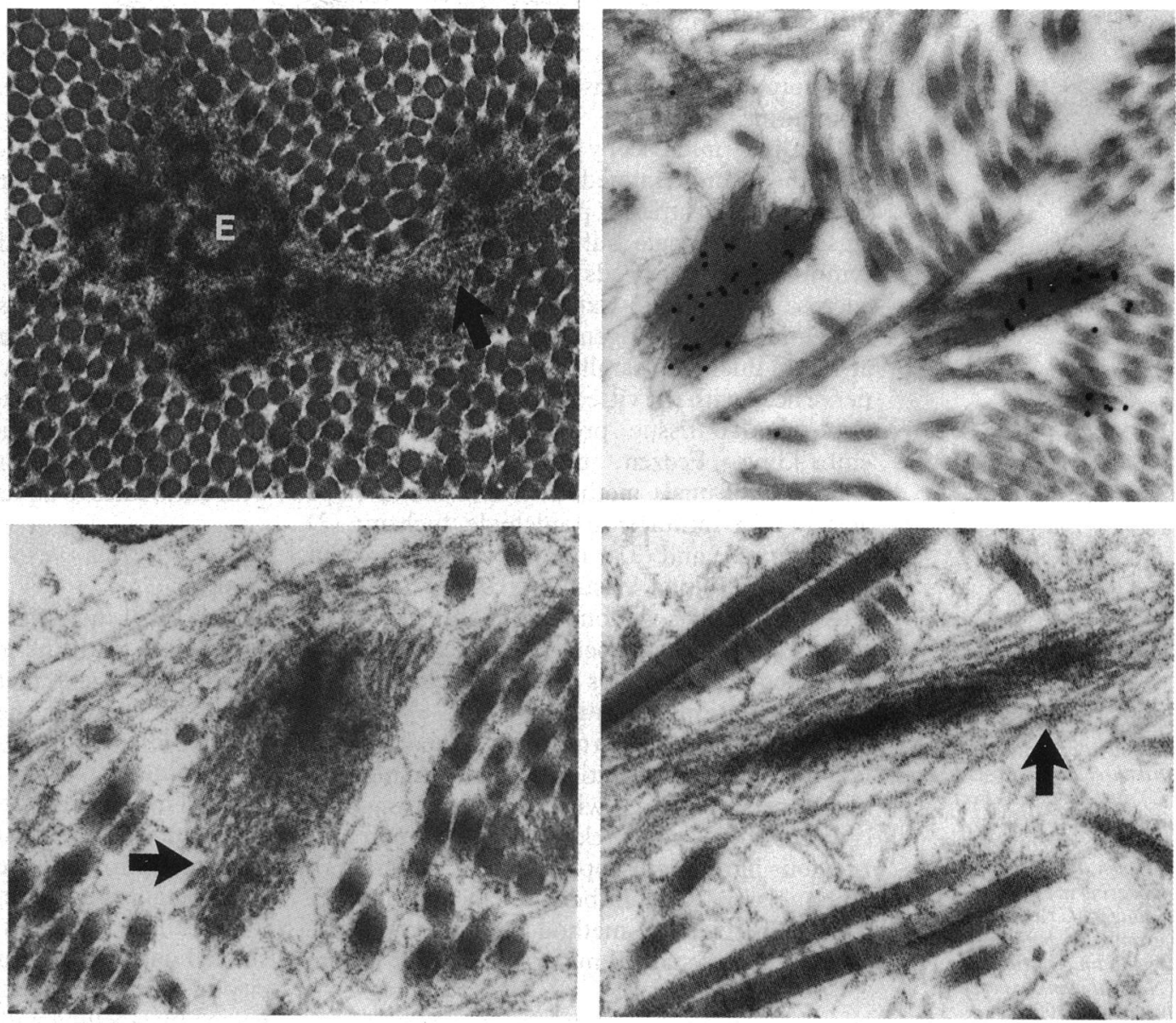

prominent change in elastin appearance was in the area(s) of nerve loss.

The number of elastin fibres in the glaucomatous eyes seemed qualitatively normal. It proved impossible to quantify elastin fibres by light microscopy. We are at present conducting quantifications by other techniques.

By electron microscopy the normal laminar

beams were surrounded by astrocytes and their basement membrane. The beam core consisted of fibrillar collagen (types I, III, and VI), elastin, glycosaminoglycans, and the laminar capillaries with their associated basement membranes (Fig 1). Even in the mildly damaged glaucomatous eyes the lamina cribrosa beam structure was disrupted, leading to a lower density of those

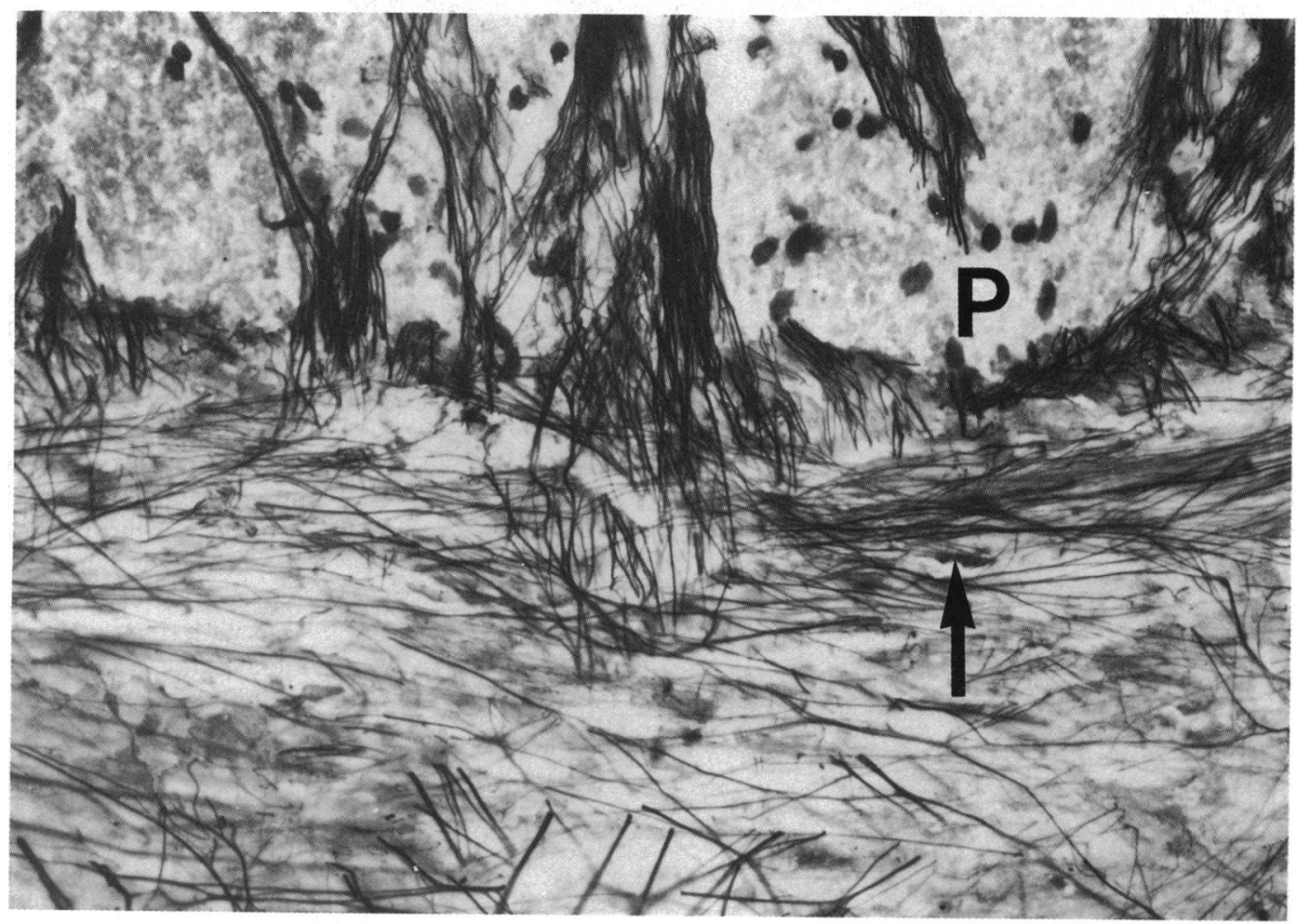

Figure 3 Zone of sclera immediately adjoining the nerve head (insertion zone). Note dense ring of elastin surrounding nerve head opening (arrow) and elastin of most peripheral lamina cribrosa beam perpendicular to the ring passing from scleral rim into nerve head between neural pores $(P)$. (Normal human eye, Luna method, frozen section, $\times 540$.) 

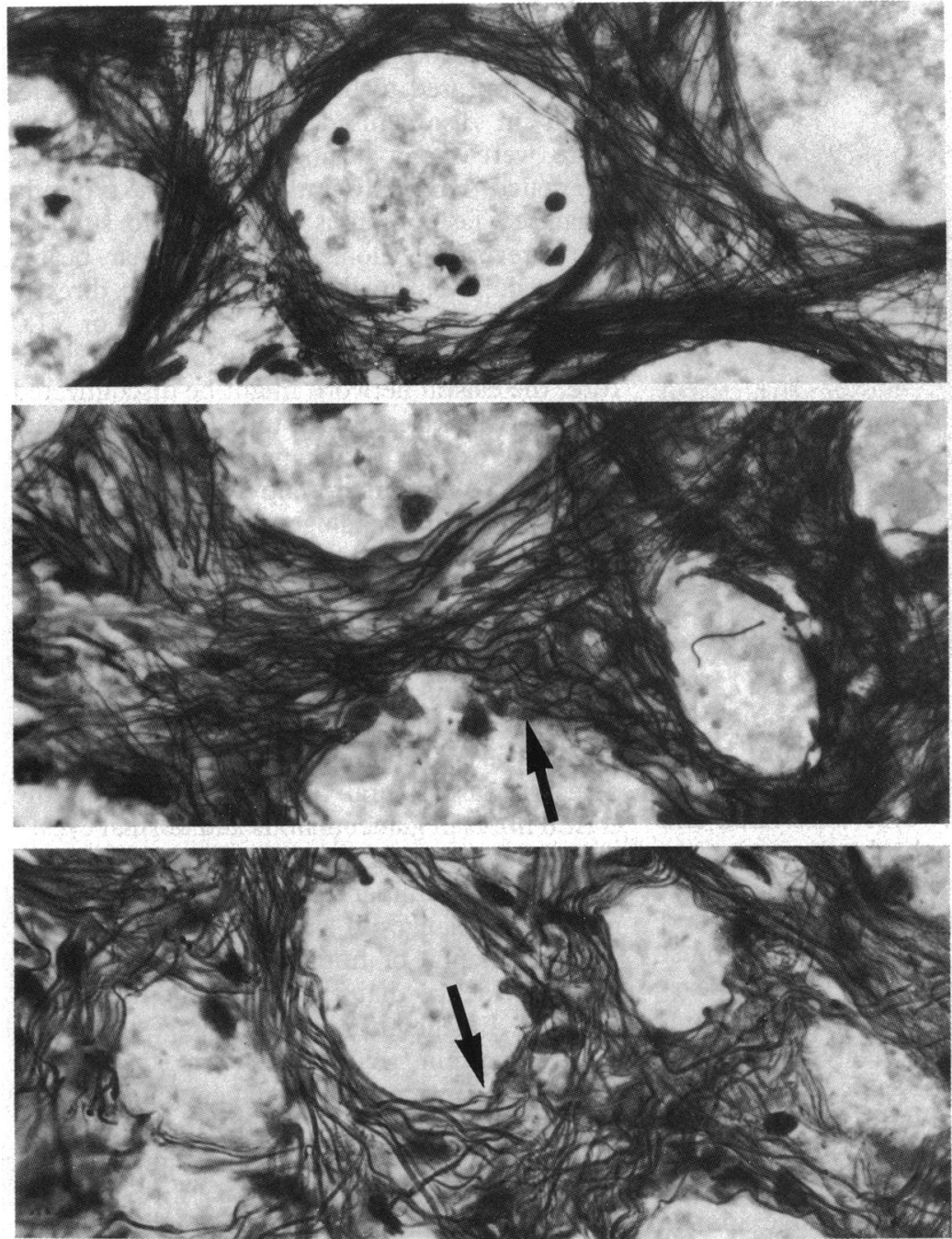

Figure 4 Light micrographs showing lamina cribrosa beams stained for elastin in nerve heads classified as normal (upper), mildly damaged (middle), and severely damaged (lower). The normal elastin appearance (upper) consists of straight elastin fibres spaced evenly across the beams. In the mildly and severely damaged glaucomatous eyes the elastin fibres are curved (arrows) rather than straight. (Human normal and glaucoma stage 2 and stage 4; Luna method, frozen sections, $\times 490$.

Figure 5 Light micrograph of elastin at scleral insertion of nerve head $(S)$. Elastin in laminar beams $(L)$ runs straight across the nerve head opening (left to right), while that in the meninges $(M)$ is parallel to the long axis of the optic nerve. The septa of the optic nerve behind the line of myelination contain elastin with various directional orientations (arrow). (Normal human eye; Luna method with epoxy resin embedding, $\times 350$.)

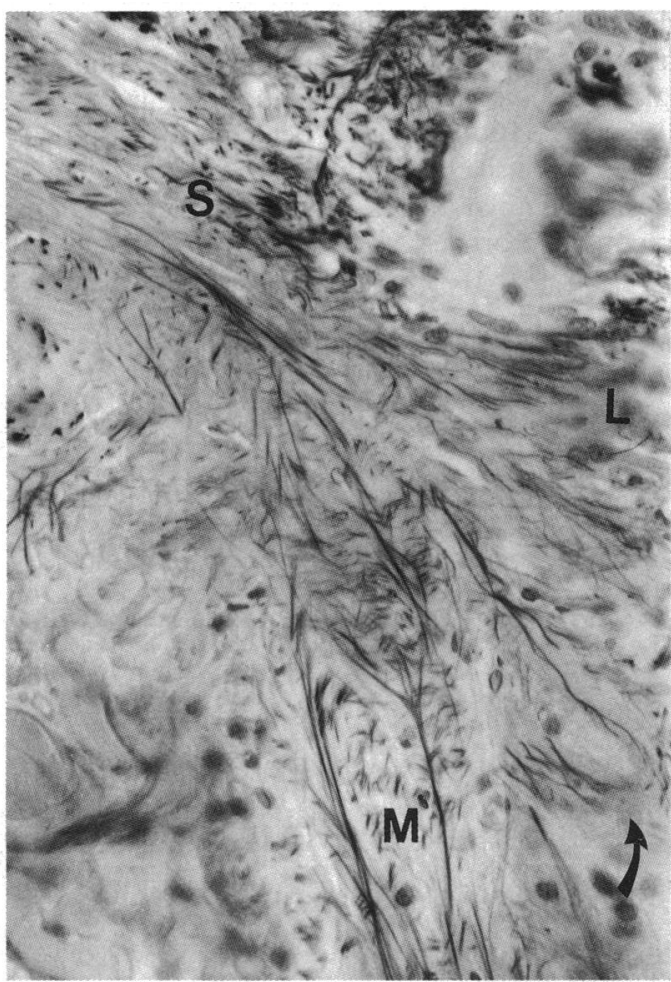

components visible by electron microscopy (Fig 6). The better preservation of the monkey eyes provided a clearer view of this change, but it was present in the human material as well. The beams underwent a movement toward the myelinated nerve from their original position. This was evident in our specimens as a loss in their astrocytic covering, and the development of electron lucent spaces between collagen and elastin fibrils (Fig 7). In the normal laminar beam collagen and elastin are tightly packed together. In the glaucomatous nerve heads, however, elastin fibres were less closely apposed to fibrillar or basement membrane collagen than in normal tissue (Figs 2,6,7). The ultrastructural substructure of the elastin fibres was not different in normal and glaucomatous eyes.

\section{Discussion}

Hernandez and coworkers ${ }^{14}$ found that the area of the sclera immediately adjacent to the lamina is rich in elastin and that there is a circumferential orientation to the molecules. They also found elastin within laminar beams, orientated preferentially across the nerve head opening. We confirm that the densest accumulation of elastin at the normal nerve head is at the insertion ring immediately surrounding the lamina, at the base of the most peripheral beams of the lamina. Here the long axis of elastin fibres is orientated circumferentially. Biomechanical analysis of a hole in a walled structure indicates that the stress at the edge of the hole might be several times that of the wall tension itself. ${ }^{15}$ Both elastin and collagen are concentrated in amount and position at this location in a way that appears to resist forces that would tend to enlarge the nerve head opening.

The long axis of elastin fibres is typically parallel to that of the collagen fibrils in the lamina. Throughout the nerve head both fibrillar species run across the lamina from one edge of the nerve head to the other. Elastin fibres are usually straight in appearance in the normal lamina. It is logical that the maximum resistance to the deformation of laminar beams is in the plane across the nerve head, compared with forces exerted from inside outward. This may explain why the most prominent change in the structure of nerve head in glaucoma is not enlargement of its diameter at the level of Bruch's membrane, ${ }^{16}$ but an anteroposterior compression of the laminar plates. ${ }^{7}$

Laboratory investigations show that the primary function of elastin is to resist tissue deformation and to return its shape to normal at low levels of stress. ${ }^{17}$ Elastin consists of complexes of a 72000 molecular weight polypeptide, ${ }^{18}$ deposited on a microfibrillar matrix (fibrillin).$^{13}$ Elastin stretches under stress and returns to its original length after release of tension. Collagen appears to function in load bearing with moderate and severe stress. ${ }^{19}$ It is capable of resistance to elongation but does not restore the original position or length of the tissue as effectively as elastin does.

In both human and monkey glaucoma we found that the elastin within the lamina cribrosa appeared wavy in outline rather than straight, as 


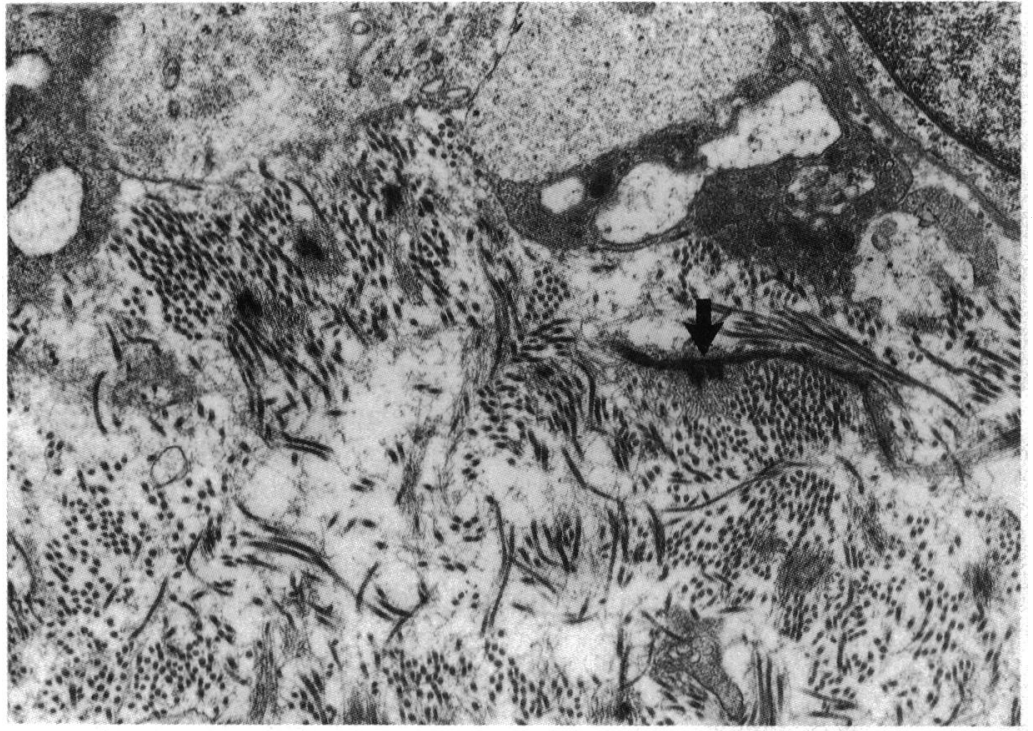

Figure 6 Lamina cribrosa beam in a monkey eye with experimental glaucoma damage. Note that by comparison with similar area in a normal monkey eye (Figure 1A) there is a dramatic disorganisation of the beam. The normal tightly packed appearance is changed to one with substantial optically empty zones among collagen fibrils and elastin molecules (arrow). (Monkey; $\times 12000$.)

Figure 7 Disorganised lamina cribrosa beam structure in experimental glaucoma monkey eye. Elastin (arrow) is found dissociated from collagen fibrils in beam with much lower than normal density of fibrillar elements. Basement membrane material (including collagen type IV) is found in several layers (asterisk) instead of the normal single layer, and is separated from its cell of origin (either capillary endothelium or astrocyte) (Monkey; $\times 16000$.)

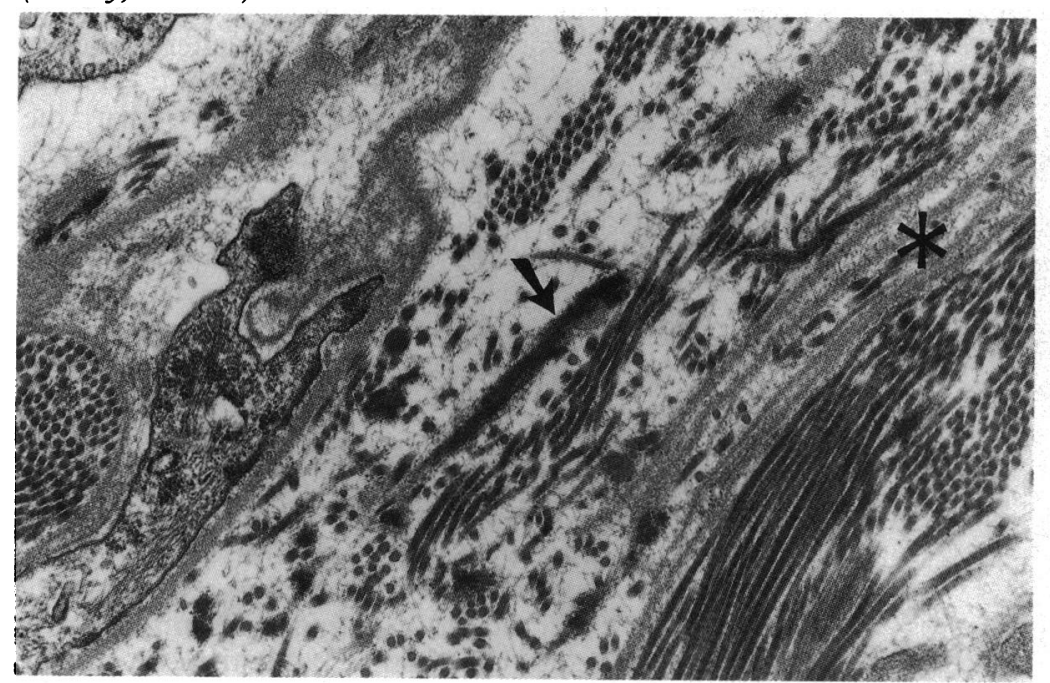

immediately next to the microfibrillar network of the elastin complex. After glaucoma injury elastin appeared to be separate from other elements in disorganised laminar beams. Since there were many beams with collagen fibrils orientated randomly rather than in typically organised fashion, it is reasonable to suppose that irreversible elongation of laminar beams occurred. ${ }^{2021}$ Normally elastin may be directly connected to collagens type I, III, IV, or VI. ${ }^{13}$ If the collagen bundles lose their normal structure, the elastin network might be disconnected from the other elements of the connective tissue. This may explain the change in elastin appearance seen by light microscopy. One possibility is that the elastin is analogous to a spring that is normally stretched in a straight line between two fixed points. When disconnected from a fixed framework, the spring assumes a curled shape. Alternatively, the wavy appearance of the elastin might have resulted from other causes. For example, the internal structure of the fibres might have been altered. However, we could not detect ultrastructural differences between the elastin fibres in glaucomatous and normal eyes.

The curled appearance of collagen within the disorganised laminar beams is only one of a number of changes in laminar connective tissue in experimental or human glaucoma. There is strong evidence that changes occur in laminar collagen. Biochemical analyses suggest a difference in the amino acid composition of collagen in normal as compared with glaucomatous eyes. ${ }^{22}$ Changes in the amount or distribution of collagen were also suggested by immunohistochemical labelling of collagens. ${ }^{2021}$ In a previous immunoelectron microscopic study ${ }^{21}$ we demonstrated reduplicated basement membrane, consisting in part of collagen type IV, within the former neural bundle areas of experimental glaucoma nerve heads. This is in a different position and has a different appearance from the curled elastin within affected laminar beams reported here.

The alterations in elastin and collagen in glaucoma may change the biomechanical behaviour of the lamina. In this regard our observations appear to be consistent with measurements of optic nerve head compliance made by Zeimer and Ogura that indicate an increased stiffness with advanced glaucomatous damage. ${ }^{23}$

This work was supported in part by PHS Research Grants EY 02120 and EY 01765 (Dr Quigley and the Wilmer Institute), by funds from National Glaucoma Research, a programme of the American Health Assistance Foundation, Rockville, Maryland, and by a Senior Investigator Award from Research to Prevent Blindness, Inc, New York.

1 Quigley HA, Addicks EM, Green WR, Maumenee AE. Optic nerve damage in human glaucoma. II. The site of injury and susceptibility to damage. Arch Ophthalmol 1981; 99: 635-49.

2 Sommer A. Intraocular pressure and glaucoma. Am $\mathcal{F}$ Ophthal mol 1989; 107: 186-8.

3 Hernandez MR, Igoe F, Neufeld AH. Extracellular matrix of the human optic nerve head. Am $\mathcal{f}$ Ophthalmol 1986;102. 139-47.

4 Morrison JC, L'Hernault NL, Jerdan JA, Quigley HA Extracellular matrix composition of the monkey optic nerve head. Invest Ophthalmol Vis Sci 1988; 29: 1141-50.

5 Hernandez MR, Iroe F Neufeld AH Cell culture of the human lamina cribrosa. Invest Ophthalmol Vis Sci 1988; 29: 78-89.

6 Anderson DR. Ultrastructure of human and monkey lamina cribrosa and optic nerve head. Arch Ophthalmol 1969; 82: 800-14. 
7 Quigley HA, Hohman RM, Addicks EM, Massof RS, Green WR. Morphologic changes in the lamina cribrosa correlated
with neural loss in open-angle glaucoma. Am $\mathcal{f}$ Ophthalmol with neural loss in

8 Repka MX, Quigley HA. The effect of age on normal human optic nerve fiber number and diameter. Ophthalmology 1989; 96: 26-31.

9 Quigley HA, Hohman RM. Laser energy levels for trabecular meshwork damage in the primate eye. Invest Ophthalmol Vis Sci 1983; 24: 1305-6.

10 Kligman LH. Luna's technique. A beautiful stain for elastin. Am 7 Dermatopathol 1981; 3: 199-205.

11 Kajikawa K, Yamaguchi T, Katsuda S, Miwa A. An improved electron stain for elastic fibers using tannic acid. $\mathcal{F}$ Electron Microsc (Tokyo) 1975; 24: 287-90.

12 Greenlee TK, Ross R, Hartman JC. The fine structure of elastic fibers. F Cell Biol 1966; 30: 59-71.

13 Sakai LY, Keene DR, Engvall E. Fibrillin, a new 350-kD glycoprotein, is a component of extracellular microfibrils. glycoprotein, is a component of
Biol 1986; 103: 2499-509.

14 Hernandez MR, Luo XX, Igoe F, Neufeld AH. Extracellular matrix of the human lamina cribrosa. Am $\mathcal{F}$ Ophthalmol 1987; 104: 567-76.

15 Greene PR. Mechanical considerations in myopia: relative effects of accommodation, convergence, intraocular pressure, and the extraocular muscles. Am $\mathcal{F}$ Optom Physiol Opt 1980; 57: 902-14.
16 Quigley HA, Brown AE, Morrison JC, Drance SM. The size and shape of the optic disc in normal human eyes. Arch and shape of the optic disc
Ophthalmol 1990; 108: 51-7.

17 Oxlund H, Manschot J, Viidik A. The role of elastin in the mechanical properties of skin. $\mathcal{F}$ Biomech 1988; 21 : 213-8.

18 Rosenbloom J. Elastin: an overview. Methods Enzymol 1987; 144: 172-96.

19 Oxlund H, Andreassen TT. The roles of hyaluronic acid, collagen, and elastin in the mechanical properties of connective tissues. F Anat 1980; 131: 611-20.

20 Morrison JC, Dorman-Pease ML, Dunkelberger GR, Quigley HA. Optic nerve head extracellular matrix in primary optic atrophy and experimental glaucoma. Arch Ophthalmol 1990; 108: $1020-4$.

21 Hernandez MR, Andrejewska WM, Neufeld AH. Changes in the extracellular matrix of the human optic nerve head in the extracellular matrix of the human optic nerve head in primary

22 Tengroth B, Ammitzboll T. Changes in the content and composition of collagen in the glaucomatous eye basis for a new hypothesis for the genesis of chronic open angle glaucoma. Acta Ophthalmol (Kbh) 1984; 62: 9991008.

23 Zeimer RC, Ogura Y. The relation between glaucomatous damage and optic nerve head mechanical compliance. Arch Ophthalmol 1989; 107: 1232-4. 